\title{
Exosomes produced from 3D cultures of umbilical cord mesenchymal stem cells in a hollow-fiber bioreactor show improved osteochondral regeneration activity
}

\author{
Litao Yan • Xing Wu
}

Received: 18 July 2019/Accepted: 21 November 2019/Published online: 9 December 2019

(C) The Author(s) 2019

\begin{abstract}
Animal and clinical studies have shown that mesenchymal stem cells (MSCs) play an important role in cartilage repair. The therapeutic effect of mesenchymal stem cells based therapies has been increasingly demonstrated to exosome-mediated paracrine secretion. Here, we investigated the cellular processes and mechanism of exosomes produced by conventional 2D culture (2D-Exos) and exosomes produced from 3D culture (3D-Exos) of umbilical MSCs (U-MSCs) in a hollowfiber bioreactor for the treatment of cartilage repair. We found that the yield of 3D-Exos was 7.5-fold higher than that of 2D-Exos. The in vitro experiments indicated that both 2D-Exos and 3D-Exos can stimulate chondrocyte proliferation, migration, and matrix synthesis, and inhibit apoptosis, with 3D-Exos exerting a stronger effect than 2D-Exos. This effect was partly attributed to the activation of transforming growth factor beta 1 and Smad2/3 signaling. The injection of 2D-Exos and 3DExos showed enhanced gross appearance and attenuated cartilage defect; however, 3D-Exos showed a superior therapeutic effect than 2D-Exos. In summary, our study
\end{abstract}

\footnotetext{
L. Yan $\cdot$ X. Wu $(\triangle)$

Department of Orthopedics, Shanghai Tenth People's Hospital, School of Medicine, Tongji University, Shanghai 200072, People's Republic of China

e-mail: wxing123@yeah.net

e-mail: sykyc@vip.163.com
}

L. Yan

e-mail: yanlitaodtc@163.com provides novel insights into the chondroprotective effects of exosomes produced from 3D culture of $U$ MSCs in a hollow-fiber bioreactor. Because of its promising biological function and high yield, 3D-Exos may become a promising therapeutic method for the treatment of cartilage defects.

Keywords Osteochondral regeneration $\cdot$ Umbilical cord mesenchymal stem cell $\cdot$ Exosome $\cdot 3 \mathrm{D}$ culture

\section{Introduction}

The adult hyaline articular cartilage is an avascular tissue that exhibits limited self-renewal capacity (Chijimatsu and Saito 2019). Traditional treatment modalities such as autografting, autologous chondrocyte implantation, and micro-fracture can temporarily alleviate symptoms, but often fail to fully restore the damaged cartilage because the regenerated tissue is unable develop an organized tissue architecture with surrounding hyaline cartilage (Gao et al. 2018).

In recent years, therapies based on stem cells have led to promising approaches for cartilage repair (Medvedeva et al. 2018; Patel et al. 2019). Laboratory and clinical studies have shown that mesenchymal stem cells (MSCs) can differentiate into chondrocytes and restore cartilage defects (Huang et al. 2016; Driessen et al. 2017; Ha et al. 2019). However, the potential of MSCs is hampered by aberrant cell phenotype, poor self-renewal ability, and reduced ability to differentiate after multiple passages when cultured in vitro. For 
transplantation of MSCs into the defect, cell vitality and viability need to be preserved; however, this requires proper handling and storage of cells and is logistically challenging (De Bari and Roelofs 2018; Dubey et al. 2018). After transplantation, the newly formed cartilage can encounter undesired hypertrophy and ossification, both of which can complicate clinical treatment (Music et al. 2018). Several studies have found that the curative effect of MSCs is more likely attributed to paracrine secretion of trophic factors rather than their differentiation into chondrocytes. Among these paracrine factors, exosomes are the most prominent extracellular vesicles that can mediate osteochondral regeneration. Exosomes are bilipid and nano-sized $(50-150 \mathrm{~nm})$ vesicles that contain nucleic acids and proteins (Toh et al. 2017). Zhang et al. (2016) have demonstrated that exosomes derived from MSCs can repair and regenerate osteochondral defects when intra-articular injected in a rat. Moreover, histological scores of the gross specimen in exosome-treated defects were significantly higher than those of control groups in vivo.

This study suggested the effectiveness of exosomes for cartilage repair, and it inspired us the cell-free therapeutic alternative to cell-based MSC therapy.

MSCs cultured in two-dimensional (2D) conventional tissue culture polystyrene flasks can result in extremely low yields, which can limit its clinical application. In our previous study, we have demonstrated that MSCs can promote the generation of cartilage and inhibit cartilage aging and osteogenesis when cultures in a $3 \mathrm{D}$ microgravity environment (Liu et al. 2016). We hypothesized that $3 \mathrm{D}$ culture might be an important factor for improved biological function. Haraszti et al. (2018) confirmed our hypothesis when they successfully obtained scalable 3D microcarrier-based cultures of MSCs. The effect of exosomes in small interfering RNA transferring was 7 -fold than 2D culture. Therefore, we speculated that exosomes produced from MSCs by 3D culture might play an important role in osteochondral regeneration. They (Haraszti et al. 2018) also found that U-MSCs yielded four times as many exosomes per cell than did MSCs from bone marrow or adipose tissue.

Until now, a commercial hollow-fiber bioreactor system has been utilized effectively for large-scale exosome production (Yan et al. 2018). In this bioreactor, cells are seeded into cylindrical fibers to simulate 3D culture. Watson et al. (2016) obtained 5-fold more exosomes using circulating medium compared to those obtained from the conventional $2 \mathrm{D}$ cell culture. This bioreactor not only allows 3D culture, but also increases the yield of exosomes. Thus, we used this bioreactor to simulate 3D culture of U-MSCs and compared the efficacy of the exosomes produced by this method with those obtained from the conventional 2D flask culture on osteochondral regeneration.

\section{Methods}

Derivation of chondrocyte

Specimens of adult articular cartilage samples were isolated from patients undergoing total knee replacement surgery. Briefly, the cartilage sample was cut into pieces about $1 \mathrm{~mm}^{3}$ and treated with $0.5 \%$ collagenase II for $4 \mathrm{~h}$. The mixture was filtered through the $100-\mu \mathrm{m}$ cell strainer and transferred to monolayer culture in DMEM-F12 (Gibco, Grand Island, NY, USA) containing $10 \%$ fetal bovine serum (FBS) and $1 \%$ penicillin/ streptomycin. After passage 3, chondrocytes were used for further in vitro experiments.

\section{D culture of U-MSCs and isolation of U-MSCs-Exos}

The primary umbilical cord mesenchymal stem cell (ASNCUC1801305, SNC, Shanghai, China) was expanded in polystyrene flasks and then seeded into a medium-sized, hollow-fiber culture cartridge. The bioreactor was purchased from FiberCell Systems (C2025, Frederick, MD, USA), and the fiber surface area was 80 $\mathrm{cm}^{2}$. The exosomes secreted by cells were gathered in the extracellular space, which had a volume of $3.9 \mathrm{~mL}$. The number of cell in $3 \mathrm{D}$ or $2 \mathrm{D}$ culture was all controlled to $1 \times 10^{7}$ (almost ten T25 culture flasks). The cell density of $3 \mathrm{D}$ culture is $2.5 \times 10^{6}$ cells $/ \mathrm{mL}$. Bioreactor conditioned medium was collected for every other day. The FBS used for culture of U-MSCs was centrifuged at $110,000 \mathrm{~g}$ overnight to deplete exosomes. The protocol for the purification of exosomes was accorded to Théry et al. (2018). Supernatants collected from bioreactor or conventional culture flask were centrifuged at same speeds ( $3000 \mathrm{~g}$ for $15 \mathrm{~min}$; 20,000 $\mathrm{g}$ for $45 \mathrm{~min}$ ). Supernatants were passed through $0.22-\mu \mathrm{m}$ filter and centrifuged for $70 \mathrm{~min}$ at $110,000 \mathrm{~g}$ to pellet exosomes. The pellets were resuspended in $5 \mathrm{~mL}$ PBS and centrifuged for another $70 \mathrm{~min}$ at $110,000 \mathrm{~g}$. All purification steps were performed at $4{ }^{\circ} \mathrm{C}$. Final pellets 
of exosomes were suspended in PBS and stored at -80

${ }^{\circ} \mathrm{C}$. The Bradford method (Bio-Rad, Hercules, USA) was used to quantify the protein concentration of the exosomes.

\section{Transmission electron microscopy}

Exosomes were suspended in PBS and dropped on the copper grid. After the pallets were dried in the air, they were fixed with $3 \%(\mathrm{w} / \mathrm{v})$ glutaraldehyde for $2 \mathrm{~h}$ and negatively stained with $2 \%$ uranyl acetate for $30 \mathrm{~s}$. The samples were observed using transmission electron microscope.

Western blot analysis

Specific markers of exosomes (CD63(ab59479), CD81(ab79559), TSG101(ab125011)) and negative marker (Calnexin (ab 92573)) were tested by western blotting. Exosomes were lysed using RIPA and heated at $95^{\circ} \mathrm{C}$ for 5 min with protein loading buffer, followed by subjecting to $12 \%$ SDS-PAGE polyacrylamide gels for $45 \mathrm{~min}$ and transferred to a PVDF membrane for $60 \mathrm{~min}$. After incubating in BSA, PVDF membranes were incubated respectively with primary antibodies at $4{ }^{\circ} \mathrm{C}$ overnight. Membranes were washed with PBST for three times, and then incubated with appropriate secondary antibody for $1 \mathrm{~h}$. All membranes were developed by enhanced chemiluminescence substrate.

\section{Nanoparticle tracking analysis}

Size distribution of exosomes was measured using ZetaView instrument. Samples were diluted 1000-fold with PBS and added to the nanopores for measurement. Readings were performed with a particle analyzer.

\section{Chondrocyte proliferation assay (Cell Counting Kit-8)}

The Cell Counting Kit- 8 assay (Beyotime, Shanghai, China) was carried out to investigate the effect of 2DExos and 3D-exos in facilitating chondrocytes proliferation. Chondrocytes $\left(2 \times 10^{3}\right.$ cells/well $)$ pretreated with $10 \mu \mathrm{g} / \mathrm{mL}$ of the two different exosomes were seeded on 96-well cell plates. The medium was changed daily for 4 days. Standard curves were constructed by measuring optical density (OD) value at $450 \mathrm{~nm}$.
Cell apoptosis assay

The chondrocyte apoptosis was detected by V-FITC/PI according to the instructions (Beyotime, Shanghai, China). Briefly, chondrocytes at density of $5 \times 10^{5}$ cells/mL were incubated with $10 \mu \mathrm{g} / \mathrm{mL} 2 \mathrm{D}$-Exos and 3D-Exos, followed by $10 \mathrm{ng} / \mathrm{mL}$ IL- $1 \beta$ challenge for 24 h. Samples were harvested and washed with PBS for three times, and then resuspended in the binding buffer. A total of $5 \mu \mathrm{L}$ Annexin V-FITC and $5 \mu \mathrm{L}$ PI were added into the suspension. After incubation away from light for $15 \mathrm{~min}$, mixtures were analyzed using flow cytometry (BD Biosciences).

Chondrocyte scratch wound assay

The migration of chondrocytes was detected by scratch wound assay. Briefly, $4 \times 10^{4}$ cells were covered the bottom of a 6-well plate. P200 pipet tip was used to scratch across the confluent monolayer of chondrocytes. The cell debris was washed with PBS. Then $1 \%$ FBS DMEM-F12 with $10 \mu \mathrm{g} / \mathrm{mL}$ exosomes was added. The condition of migration was captured at the same position after 0,24 , and $48 \mathrm{~h}$.

In vitro chondrocyte transwell migration assay

The chemotactic response of chondrocyte with various exosomes was evaluated using a transwell assay (Sigma). Chondrocyte were starved in serum-free medium for $24 \mathrm{~h}$ and seeded into the upper chambers $\left(2 \times 10^{5}\right.$ cells). Then $5 \mu \mathrm{g}$ exosomes with $500 \mu \mathrm{L} 1 \%$ FBS DMEM-F12 were added to the lower chambers. After incubating at $37^{\circ} \mathrm{C}$ for 12 and $24 \mathrm{~h}$, chondrocytes under the insert filter were fixed and then stained with $0.1 \%$ crystal violet.

Sulfated glycosaminoglycan and DNA quantification

Sulfated glycosaminoglycan was measured by Blyscan $^{\mathrm{TM}}$ Glycosaminoglycan Assay following manufacturer's instructions. Briefly, $1.0 \mathrm{~mL}$ Blyscan was added and spinned at $12,000 \mathrm{rpm}$ for $10 \mathrm{~min}$. The precipitate was mixed with dissociation reagent $(0.5$ $\mathrm{mL}$ ). All samples, standards and blanks were measured using a microplate reader at $656 \mathrm{~nm}$. DNA was measured was measured by Quant-iTTM Picogreen dsDNA Reagent and Kits. Briefly, $1.0 \mathrm{~mL}$ aqueous working solution was added and incubated for 5 minutes 
avoiding light. Samples were measured by the fluorescence microplate reader.

Western blot

After 3 days of stimulation (10 $\mu \mathrm{g} / \mathrm{mL}$ exosomes), chondrocytes were lysed using RIPA and heated at 95 ${ }^{\circ} \mathrm{C}$ for $5 \mathrm{~min}$ with protein loading buffer, followed by subjecting to $6-15 \%$ SDS-PAGE polyacrylamide gels for $45 \mathrm{~min}$ and transferred to a PVDF membrane for 60 min. After incubating in BSA, PVDF membranes were incubated respectively with primary antibodies at $4{ }^{\circ} \mathrm{C}$ overnight. Membranes were washed with PBST for three times, and then incubated with appropriate secondary antibody for $1 \mathrm{~h}$. Glyceraldehyde 3-phosphate dehydrogenase (GAPDH) was chosen as the internal reference. All membranes were developed by enhanced chemiluminescence substrate. The detail information of antibodies was in Table 1.

\section{Quantitative RT-PCR analysis}

After 3 days of stimulation ( $10 \mu \mathrm{g} / \mathrm{mL}$ exosomes), total RNA of chondrocytes was extracted by the manufacturer's instructions. PrimeScript RT-PCR kit (TaKaRa, China) was used for reversed transcription of $2 \mu \mathrm{g}$ RNA for cDNA. RT-PCR reactions were performed at the following conditions: $95{ }^{\circ} \mathrm{C}$ for $3 \mathrm{~min}, 40$ cycles of 95 ${ }^{\circ} \mathrm{C}$ for $3 \mathrm{~s}, 60{ }^{\circ} \mathrm{C}$ for $30 \mathrm{~s}$. Beta-2-microglobulin was used as an internal reference to standardize the expression of other mRNAs. The result of target gene expression was calculated by the $2^{-\Delta \Delta \mathrm{CT}}$ method expressed as the mean of three samples and presented as fold increases relative to the negative control. All primer sequences used in this study are listed in Table 2.

In vivo rabbit cartilage defect model and histological evaluation

Fifteen 2-kg New Zealand rabbits were randomly divided into three groups. The Drill bit (4-mm diameter) was used to operate cartilage defects on the trochlear grooves of the distal femurs. They were weekly respectively intra-articularly injected with: (1) $500 \mu \mathrm{L} \mathrm{PBS;} \mathrm{(2)} 500$ $\mu \mathrm{L} 2 \mathrm{D}$-Exos suspension $\left(1 \times 10^{10} \mathrm{~mL}^{-1}\right)$; (3) $500 \mu \mathrm{L}$ 3D-Exos suspension $\left(1 \times 10^{10} \mathrm{~mL}^{-1}\right)$. Four weeks later, all the animals were sacrificed with a lethal dose of anesthesia. The joints were harvested for gross appearance according to ICRS macroscopic assessment
(Table 3) from three independent blinded observers. Hematoxylin and eosin (HE), Toluidine blue (TB) and safranin-O/fast green (Saf-O) were chosen as histochemistry staining. Type II collagens were performed as immunohistochemical staining. The therapeutic effects of the repaired cartilage defect were assessed by the histologic grading scale (Wakitani et al. 1994) (Table 4) from three independent blinded observers.

Statistical analysis

The data were all showed as mean \pm standard deviation (SD). Student's $t$ test or one-way ANOVA were used for comparisons among groups. $p<0.05$ was considered as statistical significance.

\section{Results}

Characterization of U-MSCs and exosomes

U-MSCs were successfully obtained from umbilical cord Wharton's jelly. More than 95\% of U-MSCs exhibited homogeneous fibroblastic morphology after three propagations (Fig. 1a). Flow cytometric analysis revealed that a majority of U-MSCs express CD105, CD73, CD90 and are negative for CD31, CD34, CD45, and HLA-DR (Fig. 1b). Primary chondrocytes were polygonal or irregular ovoid in shape, with characteristic cobblestone morphology (Fig. 1c). Transmission electron microscopy revealed a cup-shaped morphology of the 2D-Exos (Fig. 1d left) and 3D-Exos (Fig. 1d right). Western blotting revealed that the 2D-Exos and 3DExos express exosome-associated proteins (CD63, CD81, and TSG101) as well as negative protein (Calnexin) (Fig. 1e). Nanosight analysis demonstrated that the diameter of 2D-Exos (Fig. 1f left) and 3D-Exos (Fig. 1f right) is approximately $120 \mathrm{~nm}$.

Hollow-fiber bioreactor enables high yield production of exosomes

The supernatants of U-MSCs cultured by the bioreactor or conventional 2D culture flask were purified for exosomes by centrifugation under identical conditions. The yield of 3D-Exos was 7.5-fold higher than that of 2D-Exos under identical conditions (Fig. 2a, Protein yield $=$ exosomal protein $(\mu \mathrm{g}) /$ original conditioned 
Table 1 The detailed information of antibodies

\begin{tabular}{llll}
\hline Antibody & Product code & Company & Polyclonal or monoclonal \\
\hline CD63 & ab59479 & abcam & Monoclonal \\
CD81 & ab79559 & abcam & Monoclonal \\
TSG101 & ab125011 & abcam & Monoclonal \\
Calnexin & ab92573 & abcam & Monoclonal \\
CD9 & ab92726 & abcam & Monoclonal \\
Alix & ab186429 & abcam & Monoclonal \\
GAPDH & ab181602 & abcam & Monoclonal \\
PCNA & ab92552 & abcam & Monoclonal \\
Survivin & ab76424 & abcam & Monoclonal \\
Bcl-2 & ab32124 & abcam & Monoclonal \\
Bax & ab32503 & abcam & Monoclonal \\
PDGFA & bs-0196R & Bioss & Polyclonal \\
FGF-2 & bs-0217R & Bioss & Polyclonal \\
Col II & NB600-844 & novus & Monoclonal \\
Aggrecan & WL02316 & Wanleibio & Polyclonal \\
Sox9 & ab185966 & abcam & Monoclonal \\
MMP13 & ab39012 & abcam & Polyclonal \\
ADAMTS5 & bs-3573R & Bioss & Polyclonal \\
TGFB1 & A0291 & Abclonal & Monoclonal \\
Smad2/Smad3 & A7536 & Abclonal & Polyclonal \\
phospho-Smad2/Smad3 & bs-8853R & Bioss & Polyclonal \\
Runx2 & A2851 & Abclonal & Polyclonal \\
\hline & & & \\
\hline & & & abs \\
\hline
\end{tabular}

medium $(\mathrm{mL}))$. The exosome yield $(\mu \mathrm{g})$ was determined using the Bradford assay.

The purity of exosomes was calculated from the ratio of particle to protein. The yield of 3D-Exos was approximately $1.6 \times 10^{8}$ particles/ $\mu$ g of protein, which was 6.7 fold higher than that of 2D-Exos (Fig. 2b, Particle purity $=$ number of particles/amount of exosome-associated protein $(\mu \mathrm{g}))$. Particle purity indicates the enrichment of exosome preparations.

Exosomes enhance proliferation and inhibit apoptosis of chondrocytes

To further validate our in vivo findings, we analyzed the underlying mechanism through the evaluation of both types of exosomes on the proliferation, anti-apoptosis, migration, and matrix synthesis of chondrocytes.

Cell proliferation was assessed using the CCK-8 assay and DNA concentration was determined. 2DExos and 3D-Exos were found to promote the proliferation of chondrocyte at the concentration of $10 \mu \mathrm{g} / \mathrm{mL}$.
Furthermore, 3D-Exos exhibited a much stronger effect on proliferation than 2D-Exos on day 4 ( $p<0.01$, Fig. $3 \mathrm{a}$ left). However, on day 2, there was no significant difference among the 3D-Exos, 2D-Exos, and control groups $(p>0.05)$.

The DNA concentration is proportional to the cell number. When treated with $10 \mu \mathrm{g} / \mathrm{mL}$ exosomes after $48 \mathrm{~h}$, significant differences were observed between the exosomal and control groups ( $p<0.05$, Fig. 3a right). 3D-Exos increased the amount of DNA in cells and the results were consistent with those found in the CCK- 8 assay $(p<0.05)$. The mRNA and protein expression of the genes associated with chondrocyte proliferation such as PCNA was increased within $48 \mathrm{~h}$ at 10 $\mu \mathrm{g} / \mathrm{mL}$ DNA (Fig. 3b). The induction of proliferation is associated with inhibition of apoptosis. Moreover, the protein and mRNA levels of survivin, Bcl-2, and Bax were also found to be different in both groups. Next, we assessed the IL$1 \beta$-induced anti-apoptotic effect of exosomes on chondrocytes. Compared with control group (4.6 
Table 2 Primer sequences for quantitative real-time polymerase chain reaction

\begin{tabular}{|c|c|c|}
\hline Gene & Primer & Sequence $\left(5^{\prime}-3^{\prime}\right)$ \\
\hline \multirow[t]{2}{*}{ Aggrecan } & Forward & ACCAGACTGTCAGATACCCC \\
\hline & Reverse & CATAAAAGACCTCACCCTCC \\
\hline \multirow[t]{2}{*}{ Sox9 } & Forward & GCСТCТАСТССАССТТСАСС \\
\hline & Reverse & GTAGACGGGTTGTTCCCAGT \\
\hline \multirow[t]{2}{*}{ COL II } & Forward & ATTGCCTATCTGGACGAAGC \\
\hline & Reverse & GCAGTGTACGTGAACCTGCT \\
\hline \multirow[t]{2}{*}{ MMP-13 } & Forward & GCATTGGCTGAGTGAAAGAGAC \\
\hline & Reverse & ATGATGAACGATGGACAGATGA \\
\hline \multirow[t]{2}{*}{ Runx2 } & Forward & ATGATTCGCCTCGGGGCTC \\
\hline & Reverse & GCACTCTCCGAAGGGGATCT \\
\hline \multirow[t]{2}{*}{ PCNA } & Forward & ATGATTCGCCTCGGGGCTC \\
\hline & Reverse & GCACTCTCCGAAGGGGATCT \\
\hline \multirow[t]{2}{*}{ ADAMTS5 } & Forward & ATGATTCGCCTCGGGGCTC \\
\hline & Reverse & GCACTCTCCGAAGGGGATCT \\
\hline \multirow[t]{2}{*}{ Survivin } & Forward & TTCAAGGAGCTGGAAGGCTG \\
\hline & Reverse & GCATCCGGACGAATGCTTTT \\
\hline \multirow[t]{2}{*}{ BCL2 } & Forward & TCTCATGCCAAGGGGGAAAC \\
\hline & Reverse & CAATCCTCCCCCAGTTCACC \\
\hline \multirow[t]{2}{*}{ Bax } & Forward & TCAAACCCTGCCCGAAACTT \\
\hline & Reverse & TCAGATGCCGAAGTGTGTCC \\
\hline \multirow[t]{2}{*}{ FGF-2 } & Forward & GCTGTACTGCAAAAACGGGG \\
\hline & Reverse & AGCCAGGTAACGGTTAGCAC \\
\hline \multirow[t]{2}{*}{ PDGF-AA } & Forward & GGTCGCTCCTGAAGCCAG \\
\hline & Reverse & GGAGGAGAAACAGGGAGTGC \\
\hline \multirow[t]{2}{*}{ Beta-2-microglobulin } & Forward & ATCTGAGCAGGTTGCTCCAC \\
\hline & Reverse & GGCCCTTTACACTGTGAGCC \\
\hline
\end{tabular}

chondrocytes with 2D-Exos and 3D-Exos increased the migration rate by 2 -fold to 4 -fold compared to the control group. Moreover, 3D-Exos induced an approximately 2-fold higher migration rate than 2D-Exos. The transwell migration assay further proved that 3D-Exos exhibit superior exosome-stimulating effects of migration on chondrocytes than 2D-Exos ( $p<0.01$; Fig. 4b). In addition, the mRNA and protein concentrations of PDGF-AA and FGF-2-chemotactic factors of chondrocytes - were increased within $48 \mathrm{~h}$ at $10 \mu \mathrm{g} /$ $\mathrm{mL}$ concentration (Fig. $4 \mathrm{c}$ ).

Exosomes promote matrix synthesis and phenotypic stability of chondrocytes

We next explored the ability of the exosomes to maintain phenotypic stability of chondrocytes. It is 
Table 3 International Cartilage Repair Society (ICRS) macroscopic assessment

\begin{tabular}{ll}
\hline Category & Points \\
\hline Degree of defect repair & \\
In level with surrounding cartilage & 4 \\
75\% repair of defect depth & 3 \\
50\% repair of defect depth & 2 \\
25\% repair of defect depth & 1 \\
0\% repair of defect depth & 0 \\
Integration to border zone & \\
Complete integration with surrounding cartilage & 4 \\
Demarcating border < 1 mm & 3 \\
3/4 of graft integrated, $1 / 4$ with a notable border $>$ & 2 \\
1-mm width & 1 \\
1/2 of graft integrated with surrounding cartilage, & \\
1/2 with a notable border $>1$ mm & \\
From no contact to $1 / 4$ of graft integrated with & 0 \\
surrounding cartilage & \\
Macroscopic appearance & \\
Intact smooth & \\
Fibrillated surface & \\
Small, scattered fissures, or cracks & \\
Several, small, or few but large fissure & 4 \\
Total degeneration of grafted area & 3 \\
Total maximum & 2 \\
\hline
\end{tabular}

noteworthy that the cartilage-specific markers-Col II, Sox9, Aggrecan - were highly upregulated by 3DExos; however, the hypertrophic cartilage-enriched markers ADAMTS5 and MMP 13 were downregulated by both 3D-Exos and 2D-Exos (Fig. 5a left). The mRNA levels of these proteins were consistent with the above findings (Fig. 5a right).

Along with the proliferation and passage of chondrocytes, their dedifferentiation into hypertrophic cells primarily reflects in the loss of matrix synthesis and phenotypic stability. In particular, the sulfated glycosaminoglycan (s-GAG) content was used to assess matrix synthesis of chondrocytes. At an exosomal concentration of $10 \mu \mathrm{g} / \mathrm{mL}$, the contents of s-GAG and s-GAG/DNA were significantly higher at $48 \mathrm{~h}$ in both exosomal groups than that in the PBS group (Fig. 5b). Although the s-GAG concentration of both 2D-Exos and 3D-Exos was decreased when the incubation time was extended to $72 \mathrm{~h}$, 3D-Exos showed superior ability in maintaining matrix synthesis than 2D-Exos.
Table 4 Histological grading scale for cartilage repair

\begin{tabular}{|c|c|}
\hline Category & Points \\
\hline \multicolumn{2}{|l|}{ Cell morphology } \\
\hline Hyaline cartilage & 0 \\
\hline Mostly hyaline cartilage & 1 \\
\hline Mostly fibrocartilage & 2 \\
\hline Mostly non-cartilage & 3 \\
\hline Non-cartilage only & 4 \\
\hline \multicolumn{2}{|l|}{ Matrix-staining (metachromasia) } \\
\hline Normal (compared with host adjacent cartilage) & 0 \\
\hline Slightly reduced & 1 \\
\hline Markedly reduced & 2 \\
\hline No metachromatic stain & 3 \\
\hline \multicolumn{2}{|l|}{ Surface regularity } \\
\hline Smooth $(>3 / 4)$ & 0 \\
\hline Moderate (> 1/2-3/4) & 1 \\
\hline Irregular $(1 / 4-1 / 2)$ & 2 \\
\hline Severely irregular $(<1 / 4)$ & 3 \\
\hline \multicolumn{2}{|l|}{ Thickness of cartilage } \\
\hline$>2 / 3$ & 0 \\
\hline $1 / 3-1 / 2$ & 1 \\
\hline$<1 / 3$ & 2 \\
\hline \multicolumn{2}{|l|}{ Integration of donor with host adjacent cartilage } \\
\hline Both edges integrated & 0 \\
\hline One edge integrated & 1 \\
\hline Neither edge integrated & 2 \\
\hline Total maximum & 14 \\
\hline
\end{tabular}

These results suggest that 3D-Exos is more efficient to maintain matrix synthesis and phenotypic stability of chondrocytes than 2D-Exos.

3D-Exos modulate chondrocyte functions through activation of TGF- $\beta 1$ and smad $2 / 3$ signaling pathways

The TGF- $\beta 1$ signaling pathway is the main pathway to determine whether chondrogenesis is related to proliferation, differentiation, and matrix synthesis. Therefore, we first analyzed whether the TGF- $\beta 1$-dependent $\operatorname{Smad} 2 / 3$ signaling pathway is stimulated by $3 \mathrm{D}$-Exos. The protein expression of TGF- $\beta$ 1and phospho-Smad $2 / 3$ was increased, and that of Runx2 was decreased in vitro (Fig. 5c). Therefore, we speculated that 3D-Exos might 
a

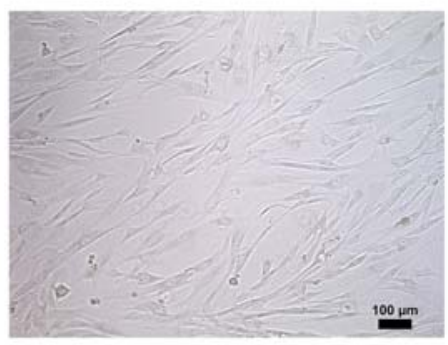

C
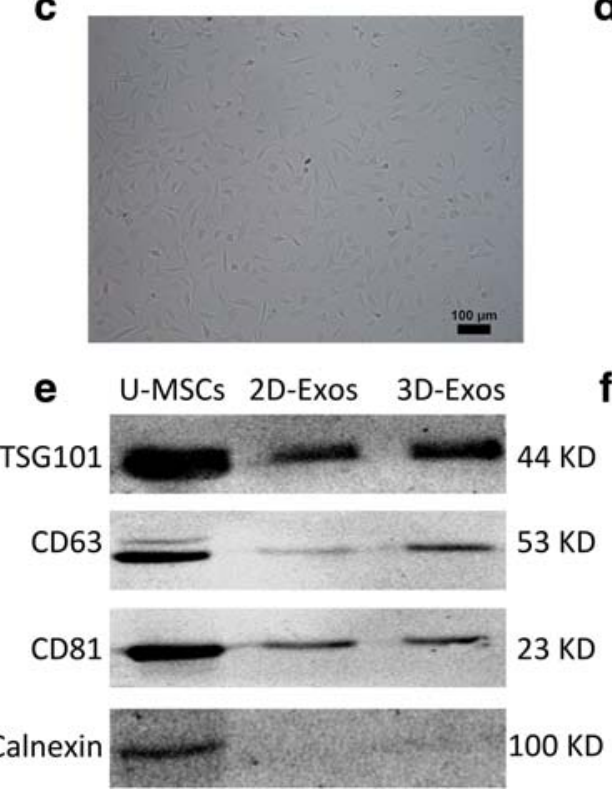

Fig. 1 Characterization of U-MSCs and exosomes. a Morphological observation of U-MSCs $(\times 100)$. b Flow cytometric analysis of umbilical cord mesenchymal positive markers, such as CD105, CD73, and CD90, and negative markers, such as CD31, CD34, CD45, and HLA-DR. c Primary human chondrocyte morphology

specifically active the TGF- $\beta 1$-dependent $\operatorname{Smad} 2 / 3$ signaling pathway to facilitate cartilage repair.

Effect of exosomes on the repair of cartilage defect

As shown in Fig. 6a, the cartilage defects treated with 3D-Exos showed more neo-tissue formation, smooth surface, and better integration with the surrounding hyaline cartilage at 4 weeks (Fig. 6a). However, the defects treated with 2D-Exos displayed poor surface regularity with structural disruptions. Moreover, the control group was the worst of all for the repair of cartilage defect as it exhibited severe tissue granulations. The ICRS macroscopic assessment scores for 3D-Exosmediated repair and 2D-Exos-mediated repair were
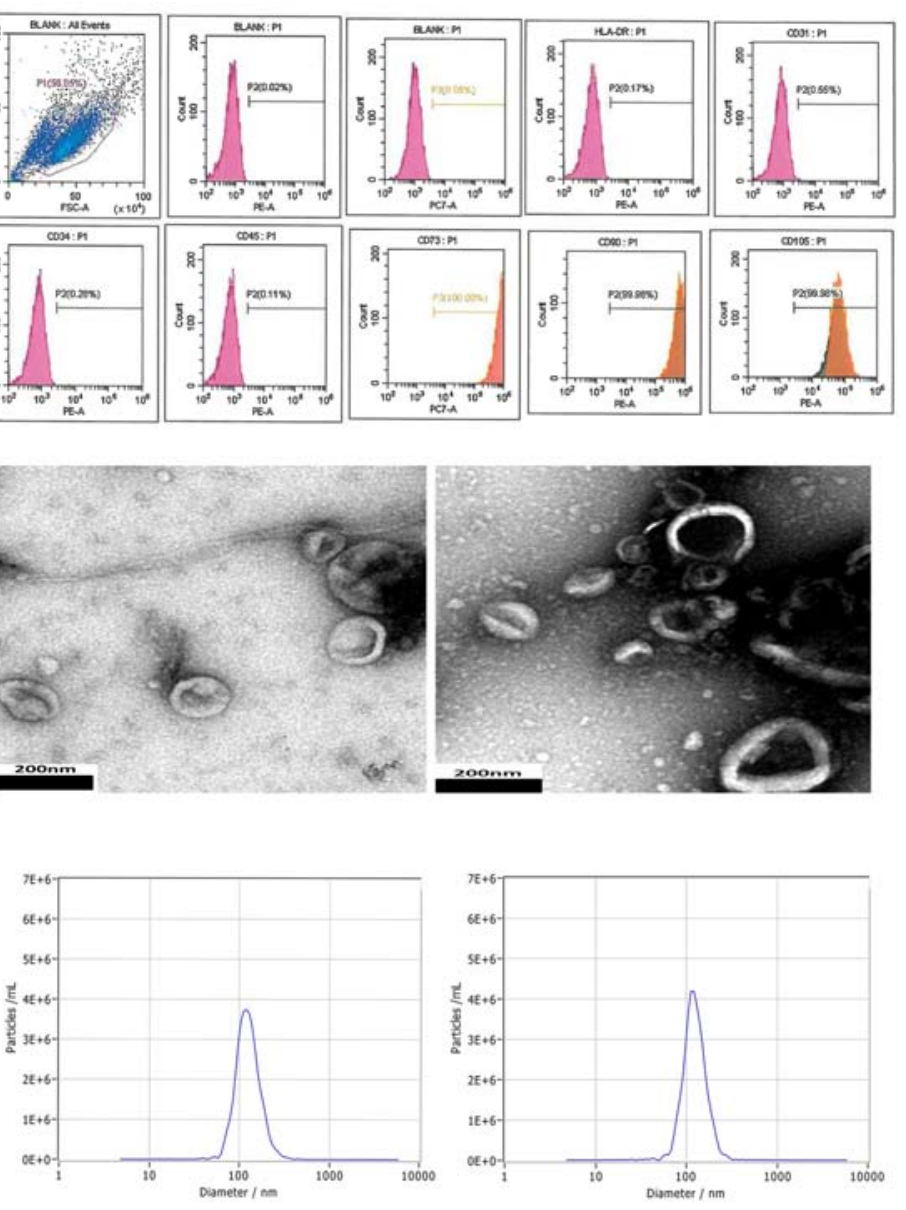

(× 100). d Morphology of 2D-Exos (left) and 3D-Exos (right) under transmission electron microscopy (scale bar $200 \mathrm{~nm}$ ). e Western blot analysis of exosome surface markers (TSG101, CD63, CD81, and calnexin). f The concentration and size distribution of 2D-Exos (left) and 3D-Exos (right) by Nanosight

significantly different. However, there was no significant difference between the 2D-Exos and control groups (Fig. 6b).

Histological assessment at 4 weeks revealed that the defects treated with both types of exosomes were partly filled with hyaline cartilage characterized by chondrocytic cells (Fig. 6c). Moreover, the defects treated with 3D-Exos showed better thickness of cartilage and surface regularity than those treated with 2D-Exos. In contrast, only fibrous tissues were found in control group, and were characterized by no metachromatic stain and severe irregularity. Consistent with these observations, the 3DExos-treated defects exhibited the highest Wakitani score (Fig. 6d). 
Fig. 2 High-yield exosomes production from hollow-fiber bioreactor. a Yield of 3D-Exos isolated by the hollow-fiber bioreactor is $\sim 7.5$-fold more than conventional flask conditioned media. Protein yield = exosomal protein $(\mu \mathrm{g}) /$ original conditioned medium (mL). b Particle purity of 3D-Exos from the hollow-fiber bioreactor is $\sim 6.7$-fold higher than conventional 2D-Exos. Particle purity $=$ the number of particles/exosomal protein $(\mu \mathrm{g})$. Plots show yield for each method and the mean \pm SD of all measurements $(* p<0.05 ; * * p<0.01)$ a

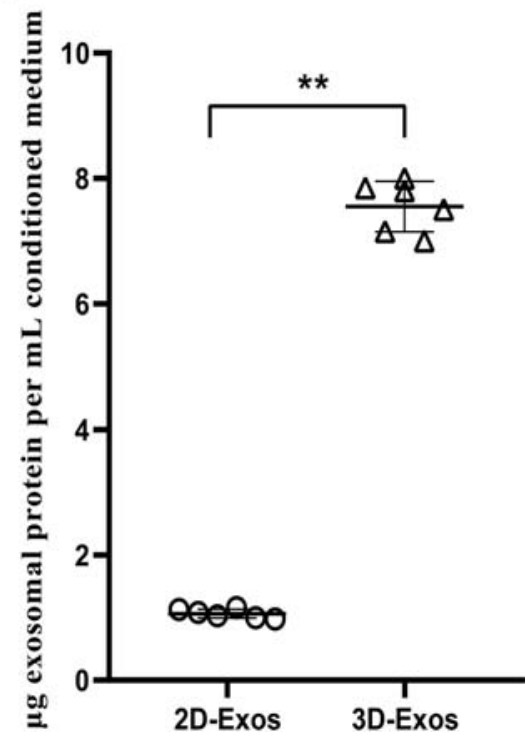

b

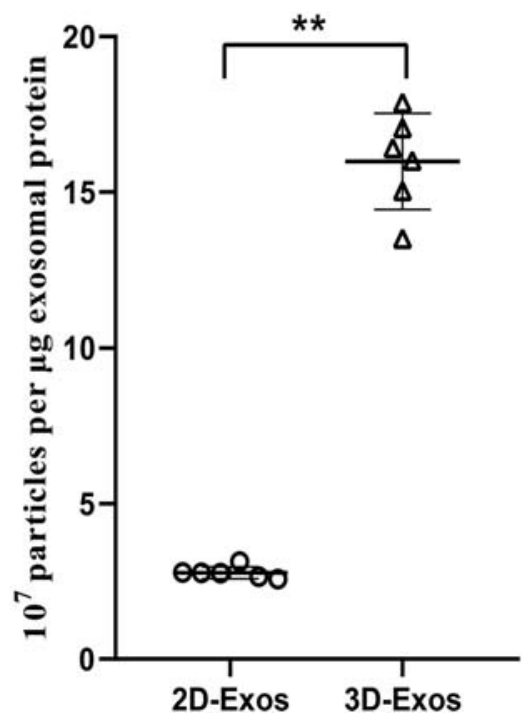

\section{Discussion}

MSC-derived exosomes have been recently identified as a novel alternative to cell-based approaches in regenerative medicine. Cosenza et al. (2017) have found that the injection of exosomes derived from MSCs into the articular cavity could protect the mice from osteoarthritis. Wang et al. (2017) also demonstrated that the cartilage destruction and matrix degradation could be alleviated by exosomes derived from human embryonic MSCs in the OA mice.

Zhang et al. $(2016,2018)$ found that MSC-derived exosomes can effectively repair osteochondral defects by enhancing cell proliferation and infiltration, inhibiting apoptosis, and regulating immune response. Furthermore, Liu et al. (2017) used an acellular tissue patch as an exosomal scaffold to promote cartilage defect repair. These studies demonstrated that MSCderived exosomes can be used as a promising, cell-free strategy for cartilage defect as an alternative to cellbased therapies. The study by Jarmalavičiūtè et al. (2015) demonstrated that exosomes derived from cells grown in a $3 \mathrm{D}$ environment show better biological functions than those derived from cells grown using the standard 2D flask culture. Thus, exosomes produced from $3 \mathrm{D}$ culture are not only more productive but also biologically active.

However, 3D culture has some disadvantages. Enzymatic digestion is required to retrieve exosomes produced within hydrogels; this may adversely affect exosomal integrity and bioactivity (Patel et al. 2018). Although the number of exosomes was increased, the intra-vesicular cargo content and function of exosomes were unknown. Thus, we performed this study to determine the ability of the hollow-fiber bioreactor to produce exosomes in 3D cultures and test their efficacy for cartilage repair.

In our current study, we innovatively compared the chondroprotective efficacy of exosomes derived from U-MSCs using different culture methods. The 3D culture of U-MSCs in a lab-scale hollow-fiber bioreactor enabled higher production yield (7.5-fold) and purity (6.7-fold) of exosomes, as shown in Fig. 2. In our study, we also found that exosomes derived from U-MSCs increased the number of chondrocytes and enhance their activity by stimulating cell proliferation, migration, and matrix synthesis, and inhibiting apoptosis. A series of experiments (Liu et al. 2017; Mao et al. 2018; Tao et al. 2018) were conducted to demonstrate that exosomes can significantly upregulate the mRNA and protein expression chondrogenesis-related factors, which are in agreement with our results. Furthermore, 3D-Exos exhibited a stronger effect than 2D-Exos to maintain the phenotypic stability of chondrocytes. Our preliminary data indicate that 3D-Exos might exert its effects through the TGF- $\beta 1$-dependent Smad2/3 signaling pathway. Several studies have demonstrated that TGF- $\beta$ s are potent inducer of chondrogenesis (Wang et al. 2016; 
a

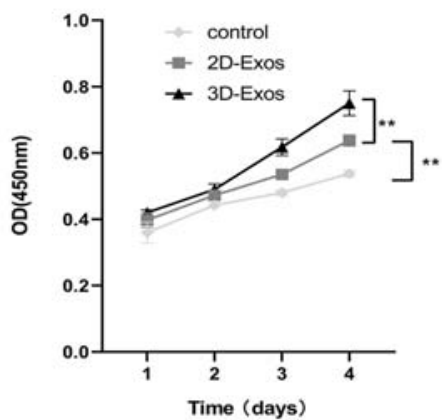

b

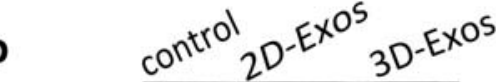

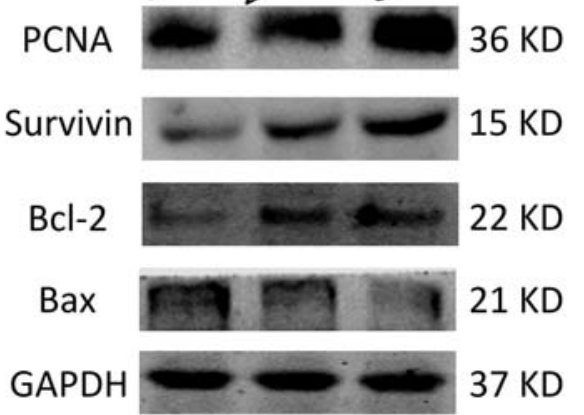

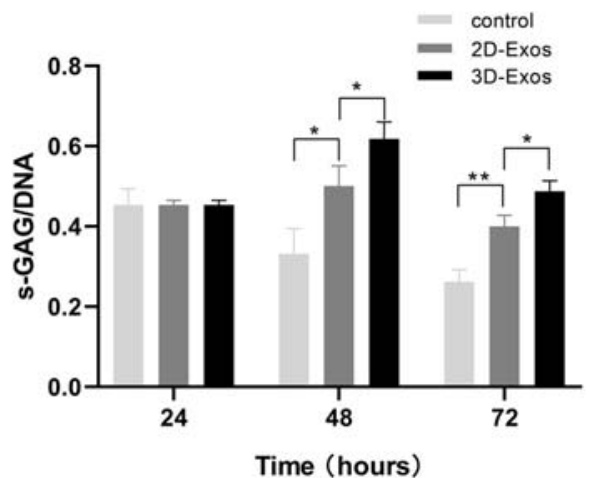

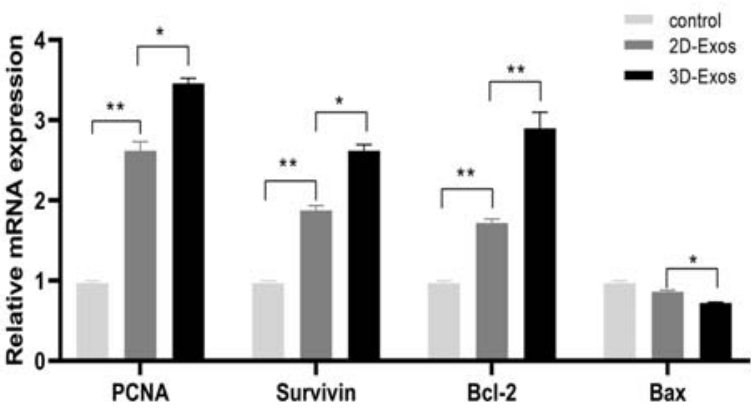

$c$
$\frac{1}{0}$
$\frac{\hat{a}}{\varepsilon}$
0
0

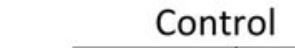

IL-1 $\beta$
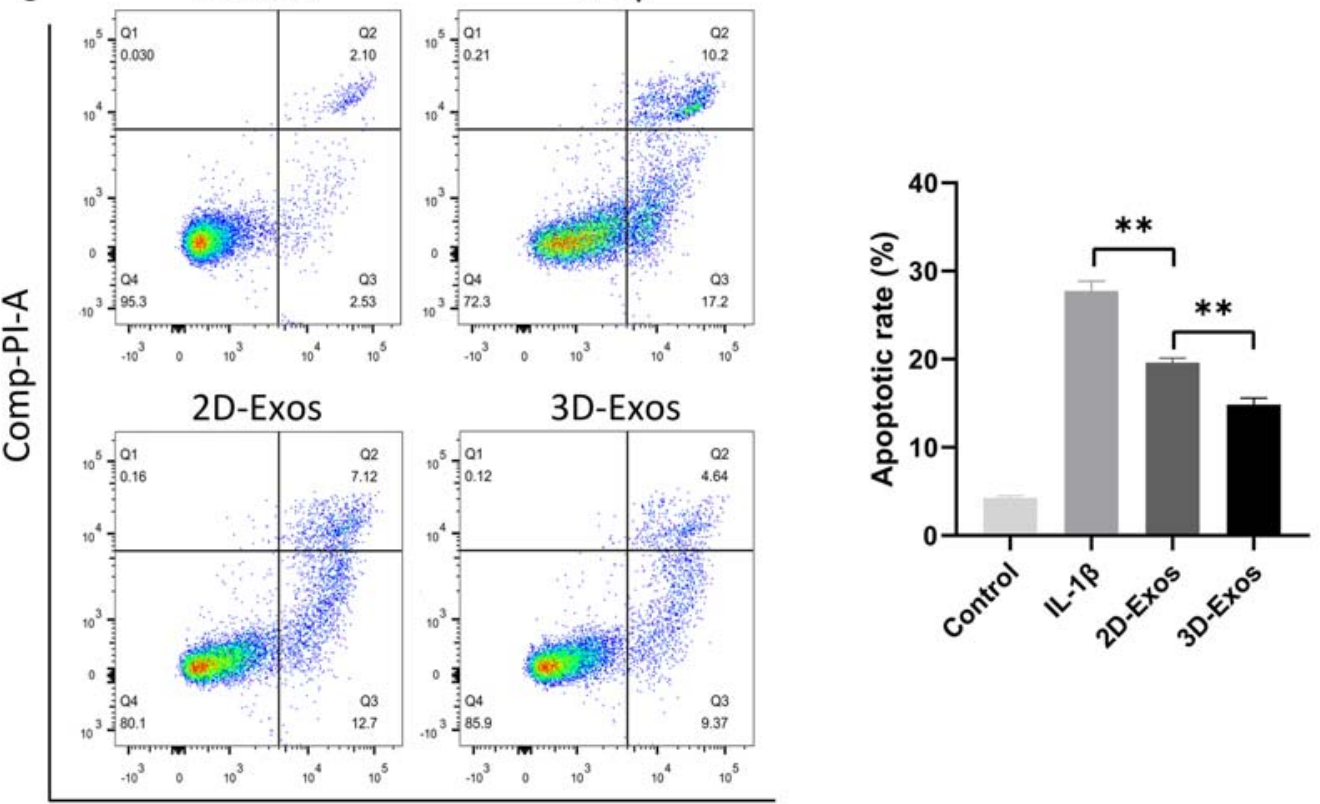

\section{Comp-FITC-A}

Fig. 3 Exosomes promote proliferation and inhibit apoptosis of chondrocytes. a The proliferation was assessed by cck- 8 assay and DNA content (right). Data represent mean \pm SEM. $* p<0.05, * * p$ $<0.01, n=5$. b The protein and mRNA level of genes associated with proliferation (PCNA) and anti-apoptosis (Survivin, Bcl-2,
Bax). c Chondrocytes were pretreated with 2D-Exos and 3DExos followed by IL-1 $\beta$ (10 ng/mL) challenge for $24 \mathrm{~h}$. Apoptosis was assessed by flow cytometry. Data represent mean \pm SEM. * $p<$ $0.05, * * p<0.01, n=3$ 
a

$\mathrm{Oh}$

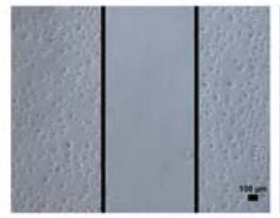

$24 \mathrm{~h}$

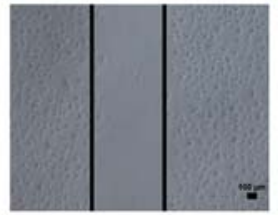

$48 \mathrm{~h}$

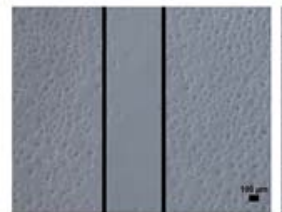

b

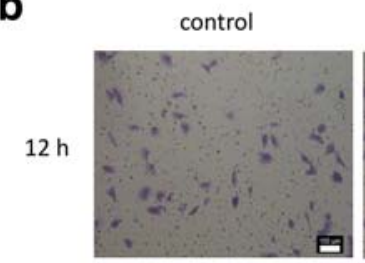

$24 \mathrm{~h}$

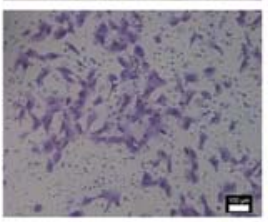

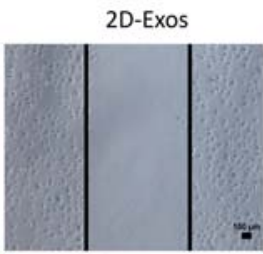
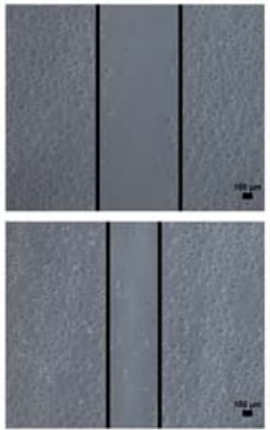

2D-Exos
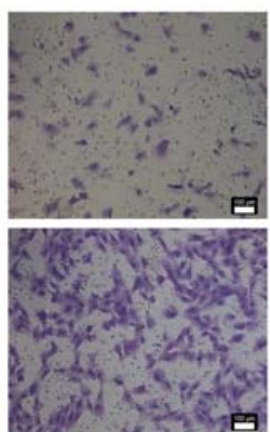

3D-Exos
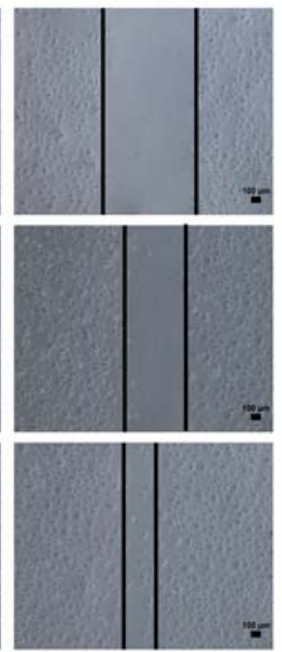

3D-Exos
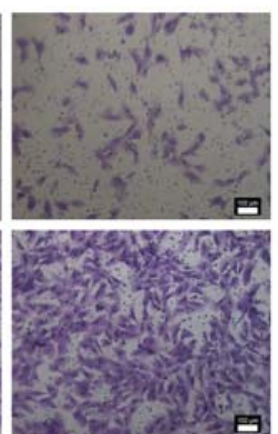

C
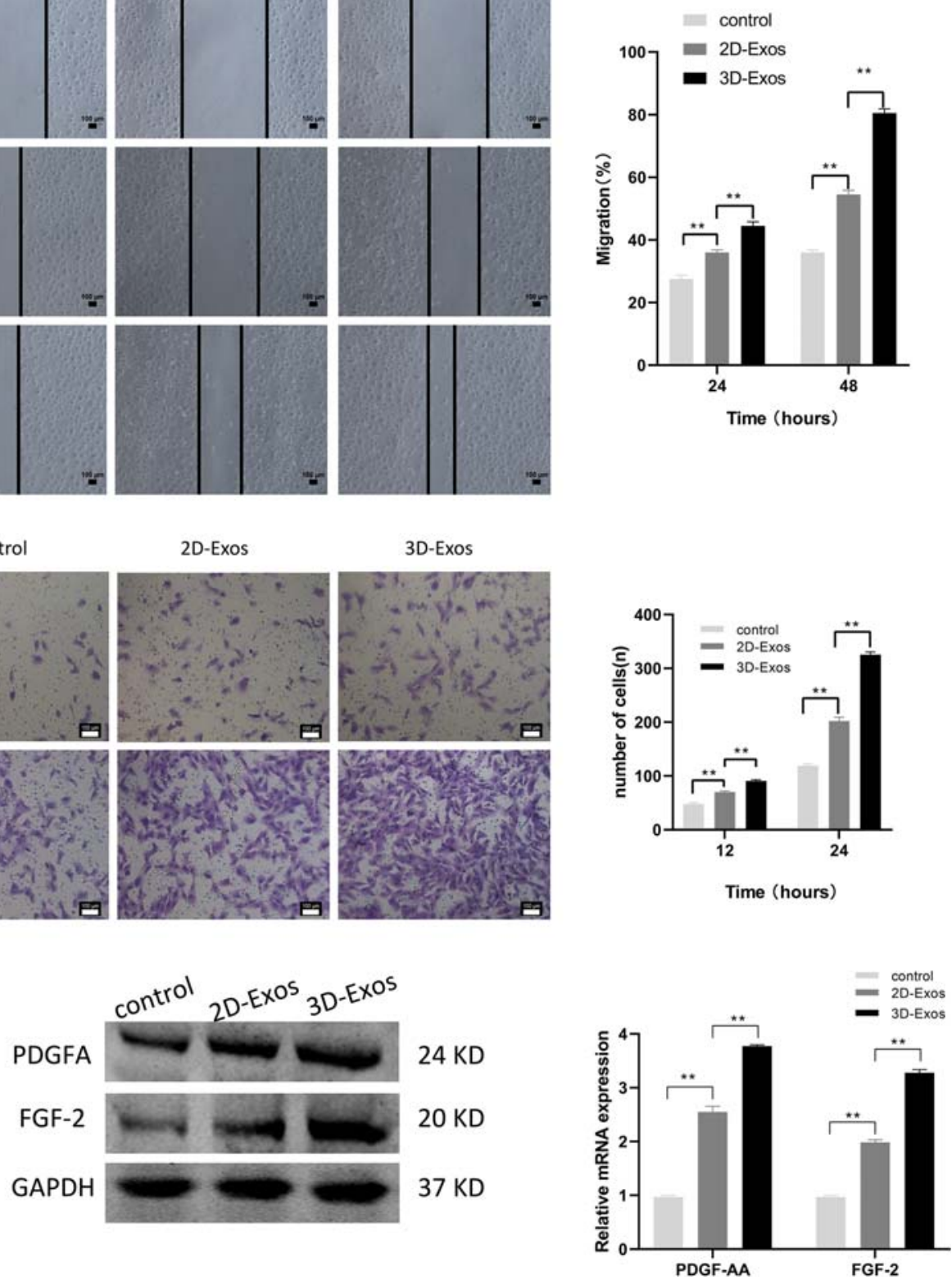

Fig. 4 Effects of 2D-Exos and 3D-Exos on migration of chondrocytes. a Light microscopy images (left) and quantitative analysis (right) of scratch wound assays. b Light microscopy images (left) and number of transmitted cells (right) in the

Ying et al. 2018; Jahr et al. 2019). The protein expression of TGF- $\beta 1$ and phosphorylated-Smad $2 / 3$ was increased and their downstream expression of Runx 2 was decreased. Therefore, we speculated that 3D-Exos might specifically activate the TGF- $\beta 1$-dependent Smad2/3 signaling pathway to facilitate cartilage repair. To our

transwell migration assay. $\mathbf{c}$ The protein (left) and mRNA level (right) of genes associated with migration (PDGF-AA, FGF-2). Data represent mean \pm SEM. $* p<0.05, * * p<0.01, n=3$

knowledge, chondrogenesis is influenced by complicated cross-talk among signaling pathways, such as Wnt, Hedgehog, BMP, and AKT/ERK (Mao et al. 2018; Ying et al. 2018; Chen et al. 2019). Further investigation is needed to picture comprehensive understanding of the pathway involved in 3D-Exos-induced chondrogenesis. 

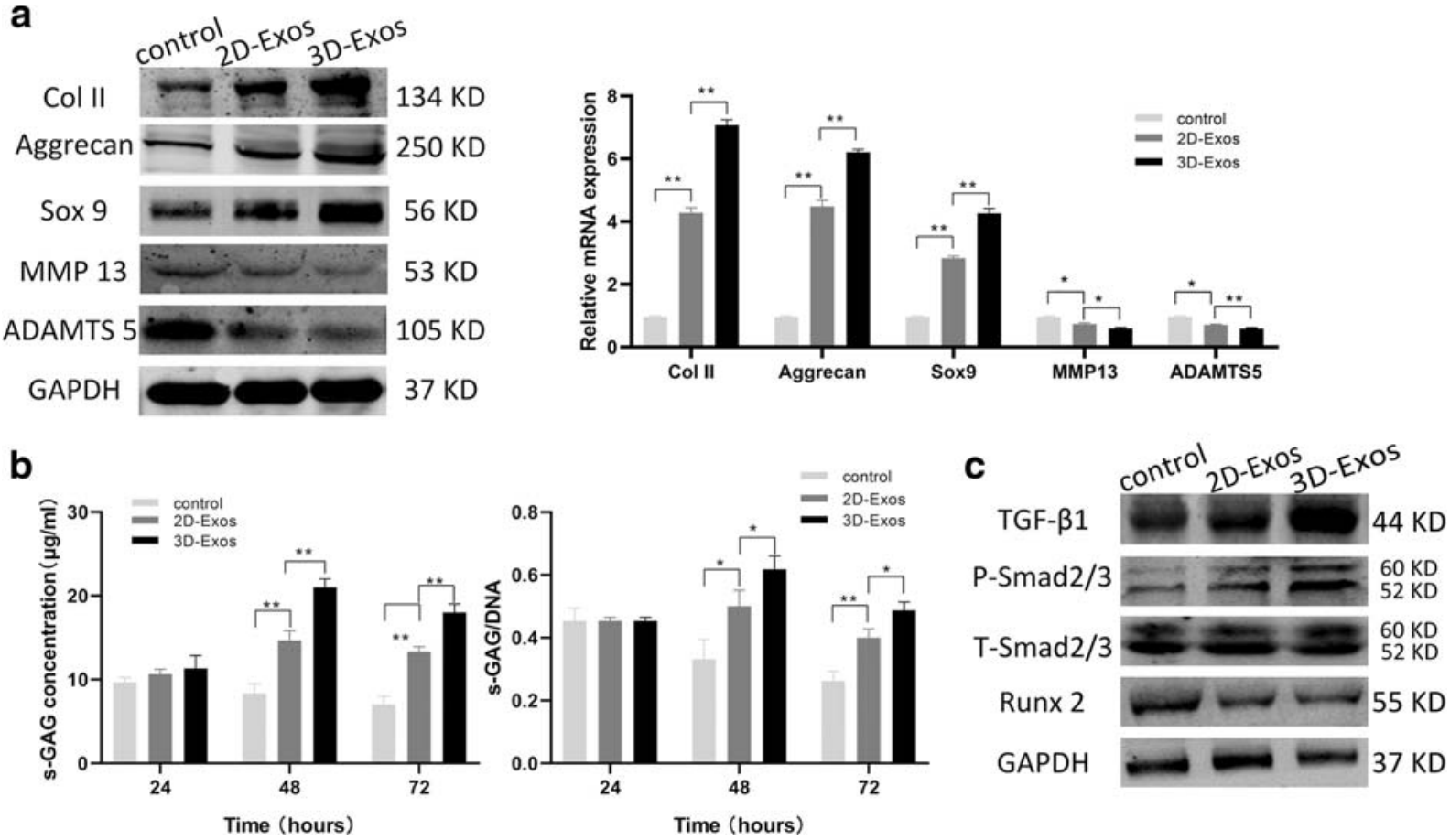

Fig. 5 Effects of 2D-Exos and 3D-Exos on matrix synthesis and the phenotypic stability of chondrocytes. a The protein and mRNA level of genes associated with matrix synthesis and chondrocytic differentiation (Col II, Aggrecan, Sox 9, MMP 13, ADMST5). b Quantitative analysis of s-GAG (left) and s-GAG/DNA (right)

when chondrocyte were stimulated by $10 \mu \mathrm{g} / \mathrm{mL}$ exosomes. c Western blot analysis of TGF- $\beta 1$, P-SMAD2/3, T-SMAD2/3, and Runx 2 of chondrocytes exposed to exosomes. Data represent mean \pm SEM. $* p<0.05, * * p<0.01, n=3$

Herein, we have demonstrated that 3D-Exos exhibits more therapeutic advantages, including higher yield and enhanced function, than 2D-Exos for cartilage repair. The enhanced yield and biological function of exosomes derived from the 3D bioreactor can be clinically applied.

Although 3D-Exos showed superior effects than 2DExos in cartilage repair in the rabbit cartilage defect model, the therapeutic outcome was inadequate for the resumption of normal function of the cartilage. One of the reasons is that the curative time lasted only for 4 weeks; however, the actual period for cartilage repair is much longer. Therefore, we will extend the in vivo experiment to 8 or 12 weeks, or even longer to better explore the kinetics of cartilage repair. Finally, multiple injections per week were the chosen mode of administration in our study. However, this is not clinically feasible and would result in increased pain to the patients. Moreover, local injection of exosomes into the joint space cannot guarantee their retention in the defect site. This would eventually lead to uncertain biodistribution of the exosomes. Therefore, exosomes embedded in scaffolds such as hydrogel tissue patch could be explored to effectively cover the cartilage defect.

Our findings indicate that culture conditions not only affect the functional status of cells but also affect exosomal activity and property. The difference in the activities and properties of 2D-Exos and 3D-Exos is related to the differences in their cargo content. We speculate that a myriad of components such as microRNAs or proteins are present in 3D-Exos, and play a crucial role in orchestrating cartilage regeneration, including promoting cell proliferation, migration, matrix synthesis, inhibiting apoptosis, and maintaining phenotypic stability of chondrocytes. However, the mechanism by which 3D-Exos exhibit superior curative effect than 2D-Exos is still unclear. Subsequent studies will be focused on comparative proteomic and RNA-seq analyses to dissect the proteins and microRNAs that are responsible for the beneficial effects. These studies will provide insights into the mechanisms underlying osteochondral regeneration of exosomes derived from $3 \mathrm{D}$ culture. 
a

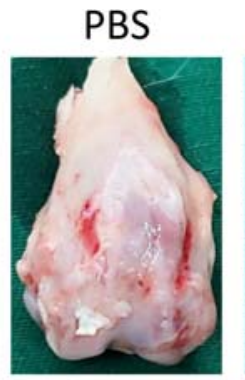

2D-Exos

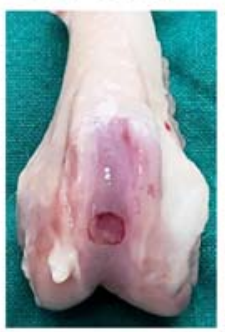

C

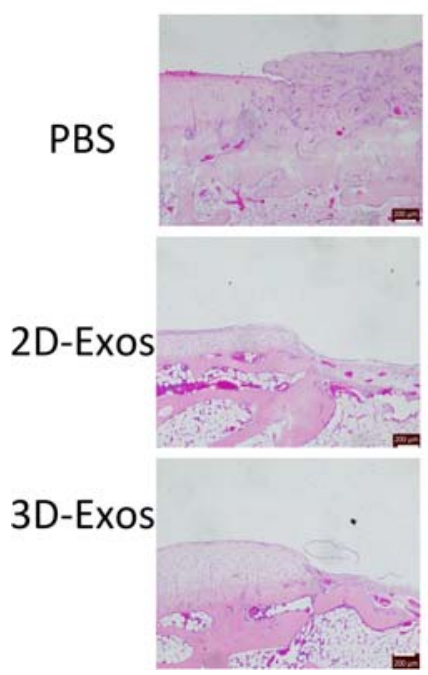

TB
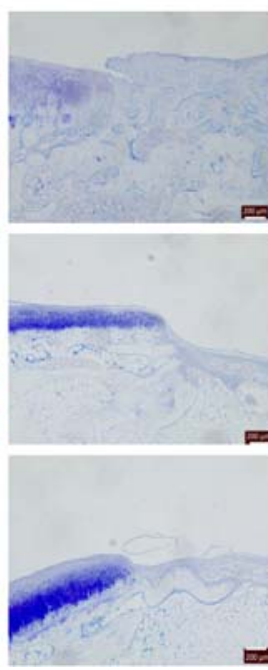

Saf-o
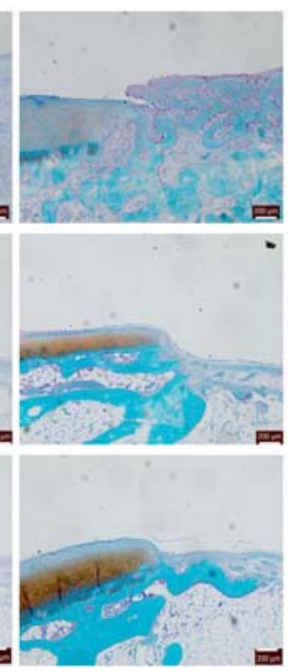

Fig. 6 Effect of exosomes on repair of cartilage defect. a Representative macroscopic images of the regenerated tissues. b ICRS macroscopic scores. Data represent mean \pm SEM. $* p<0.05$, $* * p<$ $0.01, n=5$. c Staining results of HE, TB, Saf-O, and

\section{Conclusion}

In summary, our study provides novel insights into the chondroprotective properties of exosomes derived from the 3D culture of U-MSCs in a hollow-fiber bioreactor. More specifically, 3D-Exos increased the number and enhance the function of chondrocytes by stimulating cell proliferation, migration, and matrix synthesis, and inhibiting apoptosis. Because of its enhanced biological function and yield, 3D-Exos may represent a promising therapeutic method for the treatment of cartilage defects.

Acknowledgements This project is supported by the National Natural Science Foundation of China (grant 81772324). We thank International Science Editing for editing this manuscript.

Authors' contributions Xing Wu conceived and designed the experiments. Litao Yan performed the experiments and analyzed

3D-Exos

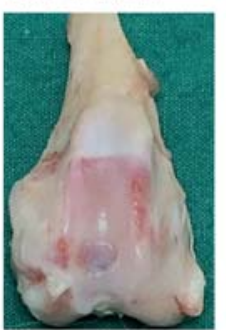

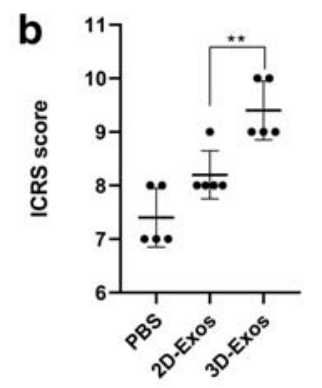

d
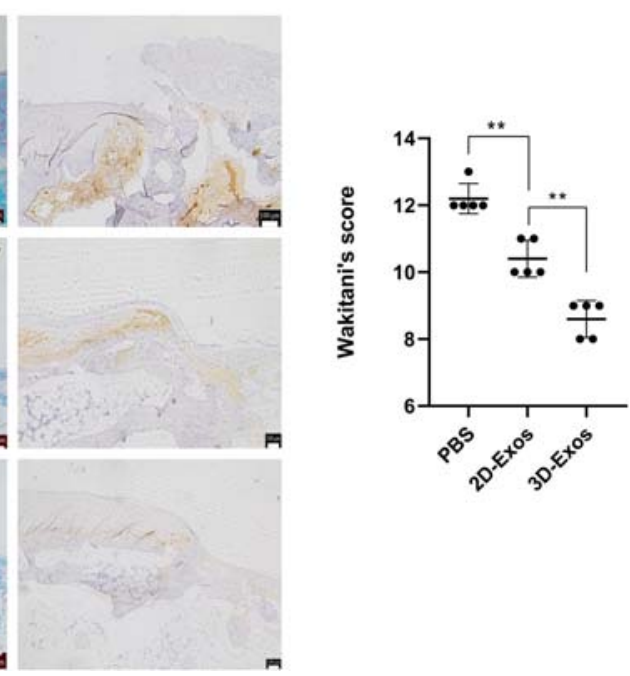

immunohistochemical staining for type II collagens. d Wakitani scores for the histological sections. Data represent mean $\pm \mathrm{SEM}$. $* p<0.05, * * p<0.01, n=5$

the data. Litao Yan wrote the paper. All authors read and approved the final manuscript.

\section{Compliance with ethical standards}

Competing interests The authors declare that they have no competing interests.

Open Access This article is licensed under a Creative Commons Attribution 4.0 International License, which permits use, sharing, adaptation, distribution and reproduction in any medium or format, as long as you give appropriate credit to the original author(s) and the source, provide a link to the Creative Commons licence, and indicate if changes were made. The images or other third party material in this article are included in the article's Creative Commons licence, unless indicated otherwise in a credit line to the material. If material is not included in the article's Creative Commons licence and your intended use is not permitted by statutory regulation or exceeds the permitted use, you will need to obtain 
permission directly from the copyright holder. To view a copy of this licence, visit http://creativecommons.org/licenses/by/4.0/.

\section{References}

Chen L, Liu G, Li W, Wu X. Chondrogenic differentiation of bone marrow-derived mesenchymal stem cells following transfection with Indian hedgehog and sonic hedgehog using a rotary cell culture system. Cell Mol Biol Lett. 2019;24:16.

Chijimatsu R, Saito T. Mechanisms of synovial joint and articular cartilage development. Cell Mol Life Sci. 2019.

Cosenza S, Ruiz M, Toupet K, Jorgensen C, Noël D. Mesenchymal stem cells derived exosomes and microparticles protect cartilage and bone from degradation in osteoarthritis. Sci Rep. 2017;7(1):16214.

De Bari C, Roelofs AJ. Stem cell-based therapeutic strategies for cartilage defects and osteoarthritis. Curr Opin Pharmacol. 2018;40:74-80.

Driessen B, Logie C, Vonk LA. Cellular reprogramming for clinical cartilage repair. Cell Biol Toxicol. 2017;33(4):329-49.

Dubey NK, Mishra VK, Dubey R, et al. Combating osteoarthritis through stem cell therapies by rejuvenating cartilage: a review. Stem Cells Int. 2018;2018:5421019.

Gao L, Goebel L, Orth P, Cucchiarini M, Madry H. Subchondral drilling for articular cartilage repair: a systematic review of translational research. Dis Model Mech. 2018;11(6).

Ha CW, Park YB, Kim SH, Lee HJ. Intra-articular mesenchymal stem cells in osteoarthritis of the knee: a systematic review of clinical outcomes and evidence of cartilage repair. Arthroscopy. 2019;35(1):277-288.e2.

Haraszti RA, Miller R, Stoppato M, et al. Exosomes produced from 3D cultures of MSCs by tangential flow filtration show higher yield and improved activity. Mol Ther. 2018.

Huang BJ, Hu JC, Athanasiou KA. Cell-based tissue engineering strategies used in the clinical repair of articular cartilage. Biomaterials. 2016;98:1-22.

Jahr H, Gunes S, Kuhn AR, Nebelung S, Pufe T. Bioreactorcontrolled physoxia regulates TGF- $\beta$ signaling to alter extracellular matrix synthesis by human chondrocytes. Int $\mathrm{J}$ Mol Sci. 2019;20(7).

Jarmalavičiūte A, Tunaitis V, Pivoraite U, Venalis A, Pivoriūnas A. Exosomes from dental pulp stem cells rescue human dopaminergic neurons from 6-hydroxy-dopamine-induced apoptosis. Cytotherapy. 2015;17(7):932-9.

Liu PC, Liu K, Liu JF, Xia K, Chen LY, Wu X. Transfection of the IHH gene into rabbit BMSCs in a simulated microgravity environment promotes chondrogenic differentiation and inhibits cartilage aging. Oncotarget. 2016;7(39):62873-85.

Liu X, Yang Y, Li Y, Niu X, Zhao B, Wang Y, et al. Integration of stem cell-derived exosomes with in situ hydrogel glue as a promising tissue patch for articular cartilage regeneration. Nanoscale. 2017;9(13):4430-8.

Mao G, Zhang Z, Hu S, Zhang Z, Chang Z, Huang Z, et al. Exosomes derived from miR-92a-3p-overexpressing human mesenchymal stem cells enhance chondrogenesis and suppress cartilage degradation via targeting WNT5A. Stem Cell Res Ther. 2018;9(1):247.
Medvedeva EV, Grebenik EA, Gornostaeva SN, et al. Repair of damaged articular cartilage: current approaches and future directions. Int J Mol Sci. 2018;19(8).

Music E, Futrega K, Doran MR. Sheep as a model for evaluating mesenchymal stem/stromal cell (MSC)-based chondral defect repair. Osteoarthr Cartil. 2018;26(6):730-40.

Patel DB, Santoro M, Born LJ, Fisher JP, Jay SM. Towards rationally designed biomanufacturing of therapeutic extracellular vesicles: impact of the bioproduction microenvironment. Biotechnol Adv. 2018;36(8):2051-9.

Patel JM, Saleh KS, Burdick JA, Mauck RL. Bioactive factors for cartilage repair and regeneration: Improving delivery, retention, and activity. Acta Biomater. 2019.

Tao SC, Guo SC, Zhang CQ. Modularized Extracellular Vesicles: The Dawn of Prospective Personalized and Precision Medicine. Adv Sci (Weinh). 2018;5(2):1700449.

Théry C, Witwer KW, Aikawa E, et al. Minimal information for studies of extracellular vesicles 2018 (MISEV2018): a position statement of the International Society for Extracellular Vesicles and update of the MISEV2014 guidelines. J Extracell Vesicles. 2018;7(1):1535750.

Toh WS, Lai RC, Hui J, Lim SK. MSC exosome as a cell-free MSC therapy for cartilage regeneration: implications for osteoarthritis treatment. Semin Cell Dev Biol. 2017;67:5664.

Wakitani S, Goto T, Pineda SJ, Young RG, Mansour JM, Caplan AI, et al. Mesenchymal cell-based repair of large, fullthickness defects of articular cartilage. J Bone Joint Surg Am. 1994;76(4):579-92.

Wang W, Song B, Anbarchian T, Shirazyan A, Sadik JE, Lyons KM. Smad2 and Smad3 regulate chondrocyte proliferation and differentiation in the growth plate. PLoS Genet. 2016;12(10):e1006352.

Wang Y, Yu D, Liu Z, et al. Exosomes from embryonic mesenchymal stem cells alleviate osteoarthritis through balancing synthesis and degradation of cartilage extracellular matrix. Stem Cell Res Ther. 2017;8(1):189.

Watson DC, Bayik D, Srivatsan A, et al. Efficient production and enhanced tumor delivery of engineered extracellular vesicles. Biomaterials. 2016;105:195-205.

Yan IK, Shukla N, Borrelli DA, Patel T. Use of a Hollow Fiber Bioreactor to Collect Extracellular Vesicles from Cells in Culture. Methods Mol Biol. 2018;1740:35-41.

Ying J, Wang P, Zhang S, Xu T, Zhang L, Dong R, et al. Transforming growth factor-betal promotes articular cartilage repair through canonical Smad and Hippo pathways in bone mesenchymal stem cells. Life Sci. 2018;192:84-90.

Zhang S, Chu WC, Lai RC, Lim SK, Hui JH, Toh WS. Exosomes derived from human embryonic mesenchymal stem cells promote osteochondral regeneration. Osteoarthr Cartil. 2016;24(12):2135-40.

Zhang S, Chuah SJ, Lai RC, Hui J, Lim SK, Toh WS. MSC exosomes mediate cartilage repair by enhancing proliferation, attenuating apoptosis and modulating immune reactivity. Biomaterials. 2018;156:16-27.

Publisher's note Springer Nature remains neutral with regard to jurisdictional claims in published maps and institutional affiliations. 